\title{
Étude exploratoire des perceptions et pratiques de médecins cliniciens enseignants engagés dans une démarche de diagnostic et de remédiation des lacunes du raisonnement clinique
}

\section{Exploratory study on the perceptions and practices of clinical teachers involved in a clinical-reasoning difficulty diagnosis and remediation process}

\section{Marie-Claude AudÉTAT ${ }^{1,2}$, Alexandre FAGUY ${ }^{3}$, André JACQUES ${ }^{4}$, Jean-Guy BlaIs ${ }^{5}$} et Bernard CHARLIN ${ }^{2}$

1 Département de médecine familiale et de médecine d'urgence, Faculté de médecine, Université de Montréal, Montréal, Canada

2 Centre de pédagogie appliquée aux sciences de la santé (CPASS), Faculté de médecine, Université de Montréal, Montréal, Canada

3 Bourse du Comité d'organisation du programme des stages d'été (COPSE), Faculté de médecine, Université de Montréal, Montréal, Canada

4 Direction de l'amélioration de l'exercice, Collège des Médecins du Québec, Montréal, Canada

5 Faculté des sciences de l'éducation, Université de Montréal, Montréal, Canada

Manuscrit reçu le 14 août 2009; commentaires éditoriaux formulés aux auteurs le 7 décembre 2009; accepté pour publication le 28 février 2011

Mots clés : Raisonnement clinique ; diagnostic des difficultés de raisonnement clinique ; remédiation
Résumé - Contexte : Si les orientations sont relativement claires dans la littérature en ce qui concerne l'enseignement du raisonnement clinique, force est de constater qu'il n'en est pas de même pour l'identification de ses lacunes et leurs remédiations. Buts : Dans cette recherche qualitative exploratoire, nous avons voulu mettre en lumière les pratiques, mais également les perceptions des cliniciens enseignants engagés dans une démarche de diagnostic et de remédiation des lacunes du raisonnement clinique. Notre cadre conceptuel s'appuie sur le modèle de Hesketh qui distingue les tâches, la façon avec laquelle ces tâches sont abordées et la perception du rôle d'enseignant. Sujets/matériel/méthode : 14 entrevues semi-structurées ont été réalisées auprès d'enseignants cliniciens travaillant dans différents contextes d'évaluation des lacunes du raisonnement clinique. Résultats : Les différentes étapes du processus pédagogique d'identification, de diagnostic et de remédiation des difficultés de raisonnement clinique se font selon un mode essentiellement intuitif. Il manque des outils spécifiques à l'évaluation du raisonnement clinique et il n'y a pas de processus pédagogique structuré et défini, ce qui diminue la précision des interventions. Ceci a un impact sur la conciliation du 
Keywords: Clinical reasoning; clinical reasoning difficulties; remediating double rôle des cliniciens enseignants : un doute quand à l'efficacité de leurs démarches pédagogiques et une tendance à se recentrer sur leur rôle de clinicien. Conclusion: Il est nécessaire d'aller «au-delà de l'intuition » pour mieux diagnostiquer les lacunes du raisonnement clinique et y remédier de façon ciblée. Afin de soutenir les cliniciens enseignants dans cette démarche, il est déterminant de développer des processus pédagogiques et des outils spécifiques.

\begin{abstract}
Context: Even though guidelines are relatively well defined in the literature regarding the teaching of clinical reasoning, it is clear that such is not the case for the identification of clinical-reasoning difficulties and their remediation. Goals: In this exploratory qualitative research, we wanted to highlight both the practices and perceptions of clinical teachers involved in a clinical-reasoning difficulty diagnosis and remediation process. Our conceptual framework builds on the Hesketh's model, which identifies tasks, the way in which these tasks are addressed and the perceived role of teacher. Subjects/material/methods: 14 semistructured interviews were conducted with clinical teachers working in different clinicalreasoning difficulty assessment contexts. Results: The different stages of the pedagogical process of clinical-difficulty identification, diagnosis and remediation are essentially intuitive. Specific clinical-reasoning assessment tools are lacking. In addition, the pedagogical process is not structured and defined, which affects the accuracy of remediation procedures. All of which impacts on reconciling clinical teachers' dual role: hesitations about the effectiveness of their teaching approaches and a tendency to focus on their role as clinicians. Conclusion: It is necessary to go "beyond intuition" to better diagnose deficiencies in clinical reasoning and propose targeted remedial plans. To support clinical teachers in this process, it is crucial to develop pedagogical processes and tools.
\end{abstract}

\section{Introduction}

Les processus de pensée et de prise de décisions qui caractérisent le raisonnement clinique sont au cœur de l'exercice professionnel. Ce sont des processus complexes, dans lesquels de multiples dimensions interagissent et qui sont caractérisés par l'incertitude et l'ambiguiité ${ }^{[1,2]}$.

10 à $15 \%$ des étudiants inscrits dans les cursus de formation médicale initiale présentent des difficultés académiques ${ }^{[3,4]}$. Différents modèles existent dans la littérature pour tenter de classifier les différents types de problèmes rencontrés; cependant, chacun s'accorde pour relever que les principales difficultés académiques sont d'ordre cognitif, parmi lesquelles figurent celles liées au raisonnement clinique ${ }^{[5-10]}$. Actuellement, ces difficultés sont identifiées la plupart du temps tardivement dans le parcours de formation ${ }^{[9-11]}$ et il n'existe que peu de descriptions de méthodes de remédiation définies $^{[12,13]}$.
L'enseignement et l'évaluation du raisonnement clinique sont donc des défis d'une grande actualité pour les enseignants cliniciens. Dans le sillage des travaux d'Elstein, puis de Kassirer, de nombreux auteurs se sont intéressés à développer des méthodes d'enseignement et d'apprentissage du raisonnement clinique qui soient davantage cohérentes avec la réalité des processus de raisonnement clinique, tels qu'ils sont mis en œuvre par les cliniciens expérimentés ${ }^{[2,14-20]}$. Ces stratégies pédagogiques consistent principalement à demander au résident d'expliciter les pensées qui sous-tendent chaque question posée au patient, mais également de justifier et prioriser chaque hypothèse diagnostique envisagée ${ }^{[15]}$. Cette perspective est intéressante parce qu'elle encourage la réflexion dans l'action et sur l'action, contribuant ainsi à développer l'expertise clinique ${ }^{[21]}$. Dans cette optique, différentes méthodes de supervision clinique, explicitement structurées et orientées sur le raisonnement clinique, ont été développées ces dernières années, 
telles que la méthode du «One Minute Preceptor ${ }^{[22,23]}$ ou encore la technique SNAPPS (Summarize history and findings, Narrow the differential ; Analyze the differential; Probe preceptor about uncertainties; Plan management; Select case-related issues for self-study) ${ }^{[24]}$.

Si les orientations sont relativement claires dans la littérature en ce qui concerne l'enseignement du raisonnement clinique, force est de constater qu'il n'en est pas de même pour l'identification de ses lacunes et leurs remédiations. En effet, plusieurs écrits mettent en évidence les nombreux obstacles rencontrés par les cliniciens enseignants. Tout d'abord, l'étendue de la littérature sur le raisonnement clinique et la diversité des approches ne facilitent pas la compréhension et l'appropriation des concepts relatifs au raisonnement clinique et à ses difficultés : même si, à ce jour, la communauté scientifique tend à se rallier autour de l'approche appelée «dual process theory ${ }^{[25-27]}$, il faut cependant noter qu'aucune théorie ni aucun modèle issus des données de recherche ne font l'unanimité ${ }^{[28]}$. De manière assez logique, puisque il n'y a pas de réel consensus au sujet du raisonnement clinique, il n'y a pas non plus d'approche structurée ni de cadre conceptuel rassembleur en ce qui concerne les difficultés de raisonnement clinique, leur identification et les stratégies pour y remédier ${ }^{[1]}$.

De façon plus globale, le contexte de l'enseignement clinique, à la merci de l'exposition clinique des étudiants et des aléas de soins, favorise un enseignement la plupart du temps informel, aléatoire et implicite $^{[29]}$ et par conséquent peu favorable à une démarche structurée et planifiée de diagnostic des difficultés.

Par ailleurs, dans ce contexte bien précis de l'enseignement professionnel, les médecins enseignants sont censés assumer deux rôles bien spécifiques : celui du clinicien, responsable du suivi médical des patients, et celui du pédagogue, responsable d'aider l'étudiant à développer ses compétences cliniques $^{[30,31]}$. À ce propos, plusieurs recherches mettent en évidence le malaise des cliniciens enseignants en ce qui concerne la démarche de diagnostic

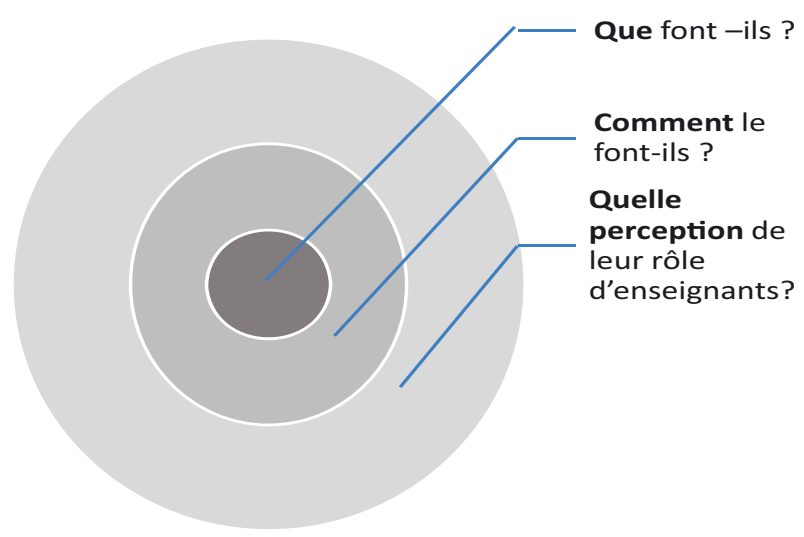

Fig. 1. Le modèle de Hesketh ${ }^{[35]}$ pour développer l'excellence en tant que clinicien enseignant.

pédagogique et de mise en place du processus de remédiation ${ }^{[33]}$, telle qu'elle est suggérée dans la littérature $^{[34]}$.

Dans cette recherche exploratoire, nous avons voulu mettre en lumière les pratiques mais également les perceptions des cliniciens enseignants engagés dans une démarche de diagnostic et de remédiation des lacunes du raisonnement clinique.

À la lumière de ces résultats, nous envisageons de développer ultérieurement des outils et des processus pédagogiques qui répondent aux éventuels besoins identifiés et qui facilitent cette démarche.

Notre cadre conceptuel s'appuie sur le modèle proposé par Hesketh et al. (figure 1) ${ }^{[35]}$, qui invite à concevoir l'excellence du clinicien enseignant selon une approche globale et qualitative, dans le cadre de laquelle trois niveaux sont identifiés : 1) les tâches effectuées (ce qu'il fait concrètement) ; 2) la façon avec laquelle il aborde ces tâches (ses perspectives théoriques, sa compréhension du problème, etc.) ; 3 ) la perception de son rôle d'enseignant et ses conséquences sur la tâche effectuée.

\section{Méthodes}

Considérant la nature exploratoire de notre recherche, nous avons choisi une méthodologie qualitative. Les données ont été constituées à partir 
d'entrevues individuelles semi-structurées permettant d'explorer en profondeur les perceptions des participants ${ }^{[36,37]}$.

\section{Préparation et déroulement des entrevues} semi-structurées

Le guide d'entrevue a été élaboré en tenant compte de la revue de la littérature et de l'expérience clinique et pédagogique de l'auteure principale qui suspectait l'absence de processus pédagogiques structurés ainsi qu'une démarche essentiellement intuitive de diagnostic des lacunes du raisonnement clinique. Le guide a été testé lors d'une entrevue pilote. Se centrant sur les pratiques et les perceptions des participants, il abordait les quatre thèmes principaux suivants : 1) la formation pédagogique des cliniciens enseignants ; 2) la démarche pédagogique et ses différentes étapes (identification-diagnosticremédiation); 3) le processus organisationnel lié à la démarche pédagogique ; 4) les difficultés et les besoins perçus par l'enseignant.

Les participants étaient par ailleurs invités à aborder toute question qu'ils jugeaient importante et qui ne figurait pas dans le guide d'entrevue. Les entrevues, d'une durée d'une heure environ, se sont déroulées sur les lieux de travail des participants.

\section{Participants}

Les participants ont été choisis selon le principe de l'échantillonnage intentionnel, décrit par Morse ${ }^{[38]}$, dans la mesure où cette façon de procéder semble être un bon moyen d'obtenir des indices et des pistes de réflexion venant alimenter une recherche exploratoire ${ }^{[36]}$. Tous sont membres du département de médecine familiale et de médecine d'urgence de l'Université de Montréal; nous avons fait ce choix dans la mesure où le département a une longue tradition pédagogique et que nous faisions l'hypothèse que cette ouverture faciliterait le recueil de données pertinentes.

Nous avons choisi des enseignants ayant une expérience différente en termes d'années d'expérience

\begin{tabular}{|l|c|}
\hline Cliniciens enseignants & $\begin{array}{c}\text { Participants } \\
N=14\end{array}$ \\
\hline Hommes & 8 \\
Femmes & 6 \\
\hline Années d'expérience en tant qu'enseignant & \\
$<10$ ans & 5 \\
$\geqslant 5$ ans & \\
\hline Implication à différentes étapes & 2 \\
du cursus professionnel des médecins & 9 \\
Niveau externat & 3 \\
Niveau de la résidence & \\
Niveau de la pratique professionnelle & \\
(Collège des Médecins du Québec) & \\
\hline
\end{tabular}

Fig. 2. Les caractéristiques des participants.

(moins de 5 ans et plus de 10 ans) afin de voir si nous trouvions des données différentes. De la même façon, nous avons identifié des enseignants travaillant dans différents contextes d'évaluation des lacunes du raisonnement clinique, tels que l'externat, la résidence, ainsi que l'évaluation des médecins en pratique dans le cadre des audits réalisés régulièrement par le Collège des médecins du Québec.

Le recrutement des participants $(N=14)$ s'est arrêté lorsque nous avons atteint la saturation de nos données. Les entrevues ont été effectuées par un étudiant de recherche (bourse COPSE de la Faculté de médecine de l'Université de Montréal), sous la supervision de la chercheure principale. Les entrevues ont été enregistrées puis retranscrites sous forme de verbatim.

\section{Analyse des résultats}

L'analyse du matériel recueilli s'est faite en deux temps. Une analyse verticale a d'abord été réalisée pour chacune des entrevues. Une analyse transversale a ensuite été effectuée afin de mettre en évidence les différentes données récurrentes au travers de l'ensemble des entrevues ainsi que les éventuelles pratiques et perceptions discordantes (de fait, très peu nombreuses). La chercheure principale et un étudiant de recherche ont procédé à l'analyse des entrevues à l'aide d'une grille de codage construite selon le cadre conceptuel choisi. Tous deux ont lu 
individuellement les entrevues, puis comparé et discuté leurs analyses et codages respectifs jusqu'à l'obtention d'un consensus (peu de désaccords). La démarche a été documentée par la tenue d'un journal de bord.

\section{Résultats}

Quatorze entrevues ont permis d'arriver à la saturation de nos données. Les résultats sont présentés en reprenant la structure de notre cadre conceptuel.

Les tâches effectuées par les cliniciens enseignants

Tous les participants soulignent qu'il ne leur faut que quelques jours de travail avec les étudiants pour soupçonner intuitivement qu'il y a un problème dans leur démarche clinique. Ces premiers « soupçons » sont souvent suivis d'une longue période (parfois plusieurs mois) au cours de laquelle peu de choses se mettent concrètement en place en terme de diagnostic pédagogique et de stratégies de remédiation, bien que la plupart des participants relèvent a posteriori la justesse et la pertinence de leurs intuitions.

Les étudiants en difficulté sont identifiés formellement la plupart du temps lors d'une réunion d'évaluation qui se fait en équipe. Au dire de tous les participants, le diagnostic pédagogique se résume dans la majorité des cas à cette identification, qui reste globale et non spécifique.

"On étiquette l'étudiant en difficulté, mais on ne le diagnostique pas. Il faudrait documenter pour que la démarche pédagogique devienne systématique. »

Les plans de remédiation sont en général établis au sein des équipes : ces plans sont relativement détaillés et précis du point de vue organisationnel, (par exemple, diminuer le nombre de patients vus par le résident pour favoriser les temps de supervision, augmenter les supervisions) de même que du point de vue du temps consacré au résident (faire davantage de supervisions directes avec le résident, donner de la rétroaction après chaque demi-journée de travail).

Cependant les plans de remédiation établis restent globaux et pas spécifiquement centrés sur les difficultés de raisonnement clinique, les enseignants ayant tendance à orienter leurs supervisions sur la gestion des situations cliniques. Quelques participants indiquent cependant connaître et utiliser des techniques spécifiques (faire expliciter au résident son raisonnement, expliciter, faire faire des lectures comparées) mais sans que cela fasse partie d'un plan structuré.

«Il y a souvent quelque chose qui va nous mettre la puce à l'oreille : ce résident là a un problème. Mais de là à poser un diagnostic de problème de raisonnement clinique, puis de formuler un plan pédagogique en conséquence. Moi je trouve qu'il y a une grosse étape, (...) je ne suis pas sûre que cette étape est faite très souvent. »

Comment les cliniciens enseignants abordent-ils ces tâches? Leurs perspectives théoriques, leur compréhension du problème

Bien que tous aient reçu une formation en ce qui concerne les étudiants en difficulté, seuls quelquesuns déclarent avoir reçu une formation spécifique sur le raisonnement clinique. La plupart n'ont jamais eu de formation sur le raisonnement clinique, ni sur les processus de diagnostic pédagogique et de remédiation.

Les participants reconnaissent la difficulté d'aller au-delà de l'intuition, puisque la perception des lacunes potentielles s'élabore peu pédagogiquement parlant. Ils observent alors qu'il y a une tendance fréquente à mettre ces lacunes sur le compte d'un manque de connaissances.

«Ça rend probablement plus flagrant quand il y a un problème de connaissance concomitant, mais de différencier les deux, c'est souvent très, très difficile. » 


\section{«Je pense que le problème de raisonnement clinique est sous-diagnostiqué encore. On a tendance à mettre beaucoup sur le dos des connaissances, ...»}

Les avis divergent en ce qui concerne l'important laps de temps qui s'écoule après l'identification intuitive des lacunes de raisonnement clinique et la mise en place de processus de remédiation : temps utile et nécessaire à l'étudiant pour s'habituer à la réalité clinique, période exploitée plus ou moins efficacement pour confirmer les éventuelles difficultés ou temps «perdu» (d'un point de vue de la remédiation) dans une formation où les différents stages se succèdent rapidement. Plusieurs attribuent ce délai à l'hésitation des cliniciens enseignants à oser établir leur diagnostic pédagogique.

«C'est un peu la difficulté : on va dire que tel résident là,... il a un problème de raisonnement clinique, souvent ils ne savent pas quoi faire après, ça s'arrête là. »

Les problèmes perçus par les participants résident essentiellement dans la définition d'un plan de remédiation pédagogique précis, ainsi que dans son application, son suivi et son évaluation dans le temps.

«Des fois, on est rendu à cinq six semaines plus tard, puis ce qui a été élaboré comme plan d'intervention s'est un peu perdu. »

La supervision directe est perçue comme un bon outil pédagogique qui permet de confirmer beaucoup plus rapidement une difficulté soupçonnée et de mieux identifier les problèmes des résidents.

Les réunions d'équipe permettent de valider les premières perceptions des enseignants, dans la mesure où les collègues témoignent de leurs propres perspectives. Il est intéressant de relever que, à ce stade, les points de vue des différents superviseurs impliqués sont rarement divergents. L'identification des lacunes de raisonnement clinique s'appuie en principe sur des processus d'évaluation définis (collecte et synthèse des évaluations quotidiennes) mais il manque cependant des outils spécifiques susceptibles de faciliter l'objectivation et la précision des lacunes potentielles. Il y a dès lors un risque, dans un contexte d'évaluation en équipe, de laisser la part belle aux processus de dynamique de groupe, tel que le déclare un participant :

"La personne qui parle le plus fort ou qui arrive avec l'exemple le plus frappant, ..., elle va vraiment colorer l'évaluation. »

La perception de leur rôle d'enseignant et les conséquences sur la tâche effectuée

Beaucoup de temps et d'énergie sont consacrés à cette démarche, mais de façon peu ciblée et peu organisée.

"La question n'est pas que les gens ne sont pas prêts à consacrer de l'énergie et du temps... On n'est pas sûrs...comment enseigner le raisonnement clinique... C'est très instinctif pour beaucoup de gens».

Par ailleurs, les participants soulignent unanimement la difficulté de concilier les deux rôles du clinicien enseignant, et ceci d'autant plus qu'ils ont affaire à un résident présentant des lacunes dans son raisonnement. Ainsi, certains ont l'impression de devoir alors se concentrer davantage sur leur rôle clinique, et ceci pour plusieurs raisons : d'une part, parce que les problèmes qu'ils identifient chez le résident ne les mettent pas en confiance et qu'ils éprouvent le besoin de vérifier, voire de "faire le travail » eux-mêmes, d'autre part parce qu'ils ont une responsabilité légale envers les patients, enfin parce qu'ils se disent plus à l'aise dans leur rôle de clinicien que dans celui de pédagogue.

L'ensemble des participants doute de l'efficacité des remédiations effectuées et disent se sentir relativement démunis dans leur rôle d'enseignant. De nombreux facteurs sont évoqués pour tenter de l'expliquer, tels que : la présence concomitante d'autres difficultés (relationnelles, manque de connaissances, manque de professionnalisme, etc.); l'organisation de la formation et sa discontinuité (stages multiples); l'organisation au sein des équipes de 
Tableau I. Résumé des résultats.

\begin{tabular}{|c|c|c|}
\hline \multicolumn{3}{|c|}{ Identification des lacunes du raisonnement clinique (RC) et remédiation } \\
\hline $\begin{array}{l}\text { Que font les cliniciens } \\
\text { enseignants? } \\
\text { - Identification rapide et } \\
\text { souvent pertinente des } \\
\text { difficultés, mais qui demeure } \\
\text { globale et intuitive. } \\
\text { - Laps de temps important } \\
\text { entre perception des lacunes } \\
\text { et remédiation. } \\
\text { - Les plans de remédiation } \\
\text { se focalisent sur des aspects } \\
\text { organisationnels, mais sont } \\
\text { peu spécifiques au RC. } \\
\text { Manque de continuité. }\end{array}$ & $\begin{array}{l}\text { Comment le font-ils? } \\
\text { - Peu d'intégration dans la } \\
\text { pratique des théories sur } \\
\text { le RC. } \\
\text { - Peu ou pas d'intégration } \\
\text { des processus pédagogiques. } \\
\text { - Les supervisions restent } \\
\text { prioritairement centrées sur } \\
\text { la gestion de la situation } \\
\text { clinique plutôt qu'orientées } \\
\text { sur une remédiation } \\
\text { pédagogique. }\end{array}$ & $\begin{array}{l}\text { Quelle perception de leur } \\
\text { rôle d'enseignant? } \\
\text { - Doute quant à leur efficacité } \\
\text { en terme de remédiation. } \\
\text { - Conflit potentiel dans la } \\
\text { conciliation des deux rôles } \\
\text { (clinicien et enseignant). } \\
\text { - Sentiment de manque de } \\
\text { maîtrise des processus } \\
\text { pédagogiques. } \\
\text { - Perception de manque } \\
\text { d'outils et de processus pour } \\
\text { soutenir la démarche } \\
\text { pédagogique. }\end{array}$ \\
\hline
\end{tabular}

superviseurs (beaucoup de superviseurs, de changement, des processus d'équipe parfois inadéquats, une mauvaise communication, etc.); la complexité du raisonnement clinique et le peu d'intégration des théories dans le contexte clinique; le manque de connaissances pédagogiques et de démarches pédagogiques systématiques; enfin le manque d'outils pédagogiques.

L'ensemble des participants relève néanmoins la nécessité d'être capable de poser un diagnostic pédagogique précis, puis de savoir plus concrètement et plus systématiquement quels moyens et outils mettre en œuvre une fois le problème identifié. Tous soulignent le besoin d'un outil d'évaluation spécifique des difficultés de raisonnement clinique, afin de pouvoir être plus systématique, mettre en mots ce qu'ils savent intuitivement et organiser leurs connaissances sur le raisonnement clinique.

«Si j'avais à résumer, un outil qui nous permettrait d'objectiver les perceptions des superviseurs, les impressions. »

\section{Discussion}

Synthèse critique des résultats

Le modèle de Hesketh nous a permis de mieux comprendre comment s'articulent les pratiques et les perceptions des cliniciens enseignants en ce qui concerne la démarche de diagnostic et de remédiation des lacunes du raisonnement clinique (tableau I).

Les résultats obtenus au niveau des tâches effectuées mettent en évidence que les cliniciens enseignants ont rapidement l'intuition de la présence de difficultés de raisonnement clinique et que cette intuition se révèle la plupart du temps pertinente. Cette perception des lacunes du raisonnement clinique demeure cependant globale et il se passe souvent beaucoup de temps avant la mise en place de remédiations. Ces remédiations sont alors prioritairement organisationnelles et peu ciblées sur le raisonnement clinique. 
Les différentes étapes du processus pédagogique d'identification, de diagnostic et de remédiation se font selon un mode essentiellement intuitif, et par conséquent peu structuré.

Le niveau relatif à la conceptualisation des tâches souligne le fait que, bien que le raisonnement clinique soit au cœur de leur profession, les cliniciens enseignants ne font preuve que d'une compréhension implicite de leurs processus de raisonnement. La littérature sur le raisonnement clinique en général est complexe et, de ce fait, peu familière à la plupart des enseignants cliniciens ; dès lors, ils font appel la plupart du temps à leur savoir pratique de cliniciens expérimentés pour guider les étudiants dans leurs apprentissages. Leurs perceptions des difficultés ne sont pas soutenues par une compréhension approfondie et explicite du développement du raisonnement clinique, ce qui contribue vraisemblablement à une perte de temps avant l'établissement d'un diagnostic pédagogique, ainsi qu'à un risque de confusion entre les problèmes de connaissances et les problèmes de raisonnement clinique.

Leurs diagnostics et plans de remédiation ne sont pas suffisamment étayés par des outils spécifiques au raisonnement clinique ni par un processus pédagogique structuré et défini, ce qui diminue la précision de leurs interventions.

Les éléments ci-dessus semblent avoir un impact sur le niveau de la perception de leur rôle d'enseignant ainsi que sur la conciliation de leur double rôle de clinicien et d'enseignant. Bien que leur motivation pour aider les étudiants soit bien présente, il apparaît néanmoins que les participants doutent sincèrement de l'efficacité de leurs démarches pédagogiques. Il est dès lors possible de faire l'hypothèse que ces doutes, d'une part ne favorisent pas la perception positive de leur rôle d'enseignant, d'autre part les invitent à se recentrer sur leur domaine d'expertise et leur rôle de clinicien.

Le mandat pédagogique des cliniciens enseignants ne doit pas être sous-estimé ou banalisé ${ }^{[29,39]}$ et les compétences nécessaires au développement de leur rôle d'enseignant devraient faire l'objet d'une réflexion approfondie. Dans cette perspective, le modèle de Hesketh, en se centrant sur les résultats observables à chacun des trois niveaux discutés précédemment, permet de dessiner non seulement un portrait des difficultés potentielles auxquelles se confrontent les enseignants dans leur tâche pédagogique, mais aussi d'élaborer un cadre de réflexion pour leur formation continue ${ }^{[35]}$.

Ainsi, les résultats de cette recherche exploratoire, nous semblent mettre en évidence la nécessité pour les cliniciens enseignants de développer une connaissance explicite approfondie des processus qui caractérisent le raisonnement clinique ; il est déterminant également de maîtriser la démarche pédagogique de diagnostic et de remédiation. Nous faisons alors l'hypothèse que cette maitrise renforcera le sentiment de compétence des cliniciens en ce qui concerne leur rôle d'enseignant et leur permettra de mieux concilier leur double rôle de clinicien et d'enseignant.

\section{Limites de la recherche}

Le choix des participants, tel que nous l'avons conçu dans cette recherche exploratoire, nous a donné l'occasion d'avoir la perspective d'enseignants cliniciens, membres du département de médecine familiale et de médecine d'urgence, exerçant dans différents contextes d'évaluation des lacunes du raisonnement clinique. L'analyse de nos données n'a pas mis en évidence de différences significatives dans leurs pratiques, ni dans leurs perceptions; il en est de même en ce qui concerne le degré d'expérience de nos participants. Une des limites de notre étude réside peut-être dans le fait que notre échantillon ne semble pas inclure des participants « extrêmes » ou «déviants », qui auraient éventuellement apporté d'autres dimensions à nos données. Nos données ont été analysées par deux chercheurs de façon indépendante, assurant ainsi une première triangulation : par faute de temps, nous n'avons pu soumettre notre analyse aux participants afin de leur faire vérifier nos résultats. Enfin, notre recherche s'est faite dans le contexte bien spécifique de l'enseignement clinique au Québec : il serait intéressant 
de conduire d'autres recherches dans des contextes cliniques différents et dans d'autres spécialités afin de vérifier la transférabilité de nos résultats.

\section{Conclusion}

Les différentes théories sur le raisonnement clinique ont tendance à rester au niveau d'un débat d'experts, demeurant ainsi compliquées pour les praticiens et peu intégrées dans le contexte clinique. Il est cependant nécessaire pour les cliniciens enseignants d'aller « au-delà de l'intuition » pour mieux comprendre et identifier comment les difficultés du raisonnement clinique se manifestent chez les étudiants ; ceci permettra alors, non seulement d'améliorer la qualité de la démarche diagnostique des difficultés, mais également d'envisager des remédiations ciblées et efficaces. Afin de soutenir les cliniciens enseignants dans cette démarche, il est déterminant de développer des processus pédagogiques et des outils spécifiques.

D'autres recherches sont nécessaires pour mieux comprendre les différents facteurs ou obstacles impliqués dans la gestion des difficultés de raisonnement clinique ainsi que leurs implications.

Considérant les risques de telles difficultés, ainsi que les impacts potentiels pour les patients, il est particulièrement important de se centrer sur ce champ d'intérêt.

\section{Contributions}

Marie-Claude Audétat a effectué la recherche documentaire, piloté la recherche et rédigé les versions initiales et finales de l'article. Alexandre Faguy, dans le cadre d'une bourse COPSE de l'Université de Montréal, a interviewé tous les superviseurs et retranscrit les données. Marie-Claude Audétat et Alexandre Faguy ont conjointement analysé les résultats et élaboré leur synthèse. André Jacques, Jean-Guy Blais et Bernard Charlin ont participé aux révisions du manuscrit.

\section{Remerciements}

L'auteur remercie le Département de médecine familiale de l'Université de Montréal pour sa confiance dans ses différents travaux ainsi que tous les cliniciens enseignants qui ont participé à la recherche.

\section{Approbation éthique}

Cette recherche exploratoire fait partie d'un projet de recherche approuvé par le Comité d'éthique de la recherche du Collège des médecins du Québec.

\section{Références}

1. Mamede S, Schmidt H, Rikers R. Diagnostic errors and reflective practice in medicine. J Eval Clin Pract 2007; 13:138-45.

2. Nendaz M, Charlin B, Leblanc V, Bordage G. Le raisonnement clinique : données issues de la recherche et implications pour l'enseignement. Pédagogie Médicale 2005;6:235-54.

3. Yates J, James D. Predicting the "strugglers": a casecontrol study of students at Nottingham University Medical School. BMJ 2006;332:1009-13.

4. Faustinella F, Orlander PR, Colletti-Mann LA, Juneja HS, Perkowski LC. Remediation strategies and students' clinical performance. Med Teach 2004;26:664-5.

5. Szumacher E, Catton P, Jones G, Bradley R, Kwan J, Cherryman F et al. Helping Learners in Difficulty - The Incidence and Effectiveness of Remedial Programmes of the Medical Radiation Sciences Programme at University of Toronto and the Michener Institute for Applied Sciences, Toronto, Ontario, Canada. Ann Acad Med Singapore 2007;36:725-34.

6. Catton P, Hutcheson H, Rothman A. An academic difficulty in postgraduate medical education: Results of remedial progress at University of Toronto. Annals RCPSC 2002;35:232-7.

7. Hunt DD, Carline J, Tonesk X, Yergan J, Siever M, Loebel J. Types of problems students encountered by clinical teachers and clerkships. Med Educ 1989;23:14-8.

8. Scott Smith C, Stevens N, Servis M. A General Framework for Approaching Residents in Difficulty. Fam Med 2007;39:331-6. 
9. Hicks PJ, Cox SM, Espey EL, Goepfert AR, Bienstock JL, Erickson SS, et al. To the point: Medical education reviewsDealing with student difficulties in the clinical setting. Am J Obstet Gynecol 2005;193:1915-22.

10. Hauer K, Teherani A, Kerr K, O'Sullivan P, Irby D. Student Performance Problems in Medical School Clinical Skills Assessments Acad Med 2007;82:S69-S72.

11. Frellsen SLMD, Baker EAMDM, Papp KKP, Durning SJMD. Medical School Policies Regarding Struggling Medical Students During the Internal Medicine Clerkships: Results of a National Survey. Acad Med 2008;83:876-81.

12. Chang A, Chou C, Hauer K. Clinical skills remedial training for medical education. Med Educ 2008;42:1118-9.

13. Saxena V, O'Sullivan P, Teherani A, Irby D, Hauer K. Remediation Techniques for Student Performance Problems After a Comprehensive Clinical Skills Assessment Acad Med 2009;84:669-76.

14. Elstein A, Shulman L, Sprafka S. Medical problem solving: an analysis of clinical reasoning. Cambridge: Harvard University Press; 1978.

15. Kassirer J. Teaching Clinical Medicine by Iterative Hypothesis Testing: Let's Preach What We Practice. N Engl J Med 1983;309:921-3.

16. Charlin B. Le raisonnement clinique, quelques données issues de la recherche. Pédagogie Médicale 2001;21:5-6.

17. Eva K. Ce que tout enseignant devrait savoir concernant le raisonnement clinique. Pédagogie Médicale 2005;6:225-34.

18. Audétat MC, Laurin S. Supervision du raisonnement clinique: Méthodes et outil pour soutenir et stimuler un raisonnement clinique efficace. Le Médecin de famille canadien 2010;56:294-96.

19. Bowen J. Educational Strategies to promote Clinical Diagnostic Reasoning. N Engl J Med 2006;355:2217-25.

20. Chamberland M. Les séances d'apprentissage du raisonnement clinique. Ann Med Interne 1998;149:479-84.

21. Schön D. The Reflective Practitioner: how Professionals Think in Action. New York: Basic Books, 1983.

22. Audétat MC, Laurin S. Clinicien et superviseur, même combat ! Le Médecin du Québec 2010;45:53-7.

23. Belleflamme M, Boulouffe C, Gérard V, de Cannière L, Vanpee D. Comment développer le raisonnement clinique des étudiants en stage à l'hôpital quand on dispose de peu de temps ? "The one-minute-preceptor". Louvain Médical 2009;128:165-9.

24. Wolpaw T, Papp K, Bordage G. Using SNAPPS to facilitate the Expression of Clinical Reasoning und Uncertainties: a Randomized Comparison Group Trial. Acad Med 2009;84:517-24.
25. Eva K. What every teacher needs to know about clinical reasoning. Med Educ 2004;39:98-106.

26. Kulatunga-Moruzi C, Brooks L, Norman G. Coordination of analytic and similarity-based processing strategies and expertise in dermatological diagnosis. Teach Learn Med 2001;13:110-6.

27. Croskerry P. A Universal Model of diagnostic Reasoning. Acad Med 2009;84:1022-8.

28. Higgs J, Jones M. Chapter 1: Clinical decision making and multiple problem spaces. In: Higgs J, Jones M (Eds.). Clinical Reasoning in the Health Professions. 3rd ed. Oxford, UK: Butterworth-Heineman Ldt, 2008.

29. Chamberland M, Hivon R. Les compétences de l'enseignant clinicien et le modèle de rôle en formation clinique. Pédagogie Médicale 2005;6:98-111.

30. Irby D. What clinical teachers need to know. Acad Med 1994;69:333-42.

31. Kilminster S, Cottrell D, Grant J, Jolly B. Effective educational and clinical supervision: AMEE Guide No 27. Med Teach 2007;29:2-19.

32. Hauer K, Teherani A, Irby D, Kerr K, O'Sullivan P. Approaches to medical student remediation after a comprehensive clinical skills examination. Med Educ 2008;42:104-12.

33. Dudek N, Marks M, Regehr G. Failure to Fail: The Perspectives of Clinical Supervisors. Acad Med 2005;80:S84-S7.

34. Steinert Y, Lewitt C. Working with the "problem" resident; guidelines for definition and intervention. Fam Med 1993;25:627-32.

35. Hesketh E, Bagnall G, Buckley E, Friedman M, Goodall E, Harden R, et al. A framework for developing excellence as a clinical educator. Med Educ 2001;35:555-64.

36. Burns N, Groves SK. Understanding Nursing Research: Building an evidence-based practice. 4th ed. Maryland Heights: Elsevier Saunders; 2007.

37. Creswell J. Qualitative Inquiry and Research Design. Thousand Oaks: Sage, 1998.

38. Morse JM. Strategies of intraproject sampling. In: Munhall PL (Ed.). Nursing research: A qualitative perspective. 4th ed. Sudbury: MA Jones and Barlett, 2007.

39. Blight J. Research in medical education at the start of the century. Med Educ 2002;36:1000-1.

Correspondance et offprints : Marie-Claude Audétat, Centre de pédagogie appliquée aux sciences de la santé (CPASS), Faculté de médecine, Université de Montréal. CP 6128, succ. Centre Ville, Montréal (QC) H3C 3J7, Canada.

Mailto : mcaudetat@sympatico.ca 